\title{
SUPERCRITICAL BEHAVIOR OF THERMODYNAMIC SYSTEMS
}

\author{
A.N. Galdina \\ Oles Honchar Dnipro National University, Dnipro, Ukraine \\ e-mail: alexandragaldina@gmail.com
}

It is known that basic stability characteristics of a system are inversely proportional to fluctuations of external parameters. Above the critical point there is a region remaining homogeneous macroscopically, but becoming microheterogeneous within an interval of thermodynamic forces. Within this interval thermodynamic coefficients of stability pass finite non-zero minima. This corresponds to the considerable growth of fluctuations and indicates the occurrence of supercritical transition of continuous kind. The limit case of such continuous phase transitions is the critical state, which is also the limit point of some first-kind transitions (the limit point of phase equilibrium curve).

In this paper we consider the relation between thermodynamic stability conditions and fluctuations of external parameters of the system. We study the behavior of a simple one-component thermodynamic system (liquid, magnet, and ferroelectric) in the supercritical region and derive the equation of the line of supercritical transition for this system.

Keywords: thermodynamic stability, critical state, supercritical phase transitions, heat capacity, coefficients of stability.

Received 07.09.2019; Received in revised form 13.10.2019; Accepted 15.10.2019

\section{Introduction}

As we know, in the critical state the system is in extreme conditions - at the boundary of thermodynamic stability. So, there is maximum development of fluctuations that causes anomalies in the behavior of thermodynamic quantities. One of the main tasks of critical state thermodynamics is to reveal the origin of these anomalies, to determine the behavior of thermodynamic quantities in the vicinity of the critical points.

In this paper we consider some aspects of the behavior of main stability characteristics for a simple system above the critical temperature. Numerous experiments for liquids [1-9] indicate that supercritical area, remaining homogeneous macroscopically, becomes microheterogeneous within an interval of thermodynamic forces. From the point of view of the thermodynamic stability theory $[10,11]$ this indicates the existence of a continuous supercritical transition between supercritical phases. This kind of transition implies that system passes through a region of lowered stability, which leads to progressively increasing fluctuations [12]. The region of great growth of energy fluctuations should not necessary coincides with the region of great increase of fluctuations of, for example, density. Therefore, curves of lowered stability for different stability coefficients need not be the same. This confirms the fact that supercritical transitions occur in the certain interval of thermodynamic forces. For magnetic system we have the same situation as for liquid: under ferromagnetic transformation the system remains one-phase and transition represents its passing through the region of lowered stability, where mainly the structural transformation from one quasiphase to another takes place $[13,14]$. Magnetic mesophase is a mixture of areas with different degrees of ordering of orbital and intrinsic moments. According to thermodynamic stability the ferromagnetic transformations are of the same mesophase origin as supercritical transitions in liquid-vapor system that shows macroscopically as similar run of the curves for stability determinant and coefficients.

\section{The equation of line of supercritical transitions for thermodynamic systems}

The kind of phase transition is known to be determined by the behavior of principal characteristics of thermodynamic stability of the system: the determinant of stability $D=\partial(T, X) / \partial(S, x)$ (Jacobian consisting of derivatives of thermodynamic forces $X$ with respect to thermodynamic coordinates $x$ ), and the coefficients of stability-derivatives of 
thermodynamic forces with respect to corresponding coordinates at constant other forces or coordinates (the isodynamic quantities $(\partial T / \partial S)_{X},(\partial X / \partial x)_{T}$ or the adiabatic quantities $\left.(\partial T / \partial S)_{x},(\partial X / \partial x)_{S}\right)$. These characteristics are inversely proportional to the fluctuations of the external parameters of the system, which is the primary cause of the phase transition. During the transitions of continuous kind the coefficients of stability and $D$ pass through finite minima. It corresponds to the fluctuation growth. The locus curve of these minima is the curve of the transitions of continuous kind (the curve of supercritical transitions, the quasispinodal) [12].

It should be noted that for different coefficients of stability, the lowered stability curves may not coincide. Thus, we use as a basis the line of lowered stability for $D$, which contains all the equilibrium characteristics of the system and therefore most completely describes its stability.

The threshold case of supercritical transitions, when fluctuations in the system become maximum, $D$ and coefficients of stability pass through the zero minima, is called a critical state. The critical point is the end-point for some first-order transitions (the finite jumps of $D$ and stability coefficients are intrinsic to the first-order transitions).

Let us consider this problem in detail basing on the quasi-thermodynamic theory of fluctuations $[15,16]$. It follows from the above that the point of the supercritical phase transition - the point of lowered stability - must be the extreme point of fluctuations. For example, according to the first Gibbs lemma $(\partial T / \partial S)_{x}=k T^{3} / \overline{(\Delta H)^{2}}$ the condition

$$
d \overline{(\Delta H)^{2}}=0
$$

corresponds to the minimum of the thermal adiabatic stability coefficient $(\partial T / \partial S)_{x}$ ( $H$ is energy of the system). Considering this condition in the case of system affected by temperature $T$ and pressure $P$, we obtain a line of lowered stability - the minimum locus of $(\partial T / \partial S)_{V}$. At the critical point $(\partial T / \partial S)_{V}$ passes through a zero minimum. Above the critical point $(\partial T / \partial S)_{V}$ passes through non-zero finite minima which depth decreases with distance from the critical point.

The probability of an arbitrary state of entire system (subsystem + environment) according to the Boltzmann principle is related to the total entropy of the system $S_{\text {tot }}$ by the relationship $w \sim e^{S_{\text {tot }}{ }^{\prime k}}$. From the standpoint of fluctuations study, it can be rewritten in the form

$$
w=A e^{S_{\mathrm{tot}} / k}=A e^{\left(\Delta S_{0}+\Delta S\right) / k}
$$

where $\Delta S_{0}$ is change in entropy of environment and $\Delta S$ is change in entropy of the system compared to their values in an equilibrium state, $k$ is Boltzmann constant.

In the case of constant number of particles $N=$ const, the entropy change of environment can be represented as

$$
\Delta S_{0}=\frac{\Delta U_{0}+P_{0} \Delta V_{0}}{T_{0}}=-\frac{\Delta U+P_{0} \Delta V}{T_{0}} .
$$

The possibility of such an expression is justified by the fact that we are interested in fluctuations that occur in the system. So, the environment, because of its large size, we 
consider as equilibrium, that is the gradients of its parameters can occur only in a thin surface layer at the boundary with the subsystem. For the quantity in the exponent in (2) we get the expression $\left(T_{0} \Delta S-P_{0} \Delta V-\Delta U\right) / k T$, where $U$ is internal energy of the system.

Considering the small fluctuations, let us expand $\Delta U$ into a series in terms of powers of increments of arguments $\Delta S, \Delta V$ up to the second-order accuracy terms [17]

$$
\Delta U=\left(\frac{\partial U}{\partial S}\right)_{0} \Delta S+\left(\frac{\partial U}{\partial V}\right)_{0} \Delta V+\frac{1}{2}\left[\left(\frac{\partial^{2} U}{\partial S^{2}}\right)_{0} \Delta S^{2}+2 \frac{\partial^{2} U}{\partial S \partial V}{ }_{0} \Delta S \Delta V+\left(\frac{\partial^{2} U}{\partial V^{2}}\right)_{0} \Delta V^{2}\right],
$$

where all derivatives of internal energy are taken in the equilibrium state. Taking account of $(\partial U / \partial S)_{0}=T_{0}$ and $(\partial U / \partial V)_{0}=-P_{0}$, let us make sure that the terms linear in $\Delta S, \Delta V$ in the exponent of formula (2) cancel each other and this exponent equals

$$
-\frac{\left(\frac{\partial T}{\partial S}\right)_{0} \Delta S^{2}+\left(\frac{\partial T}{\partial V}\right)_{0} \Delta S \Delta V-\left(\frac{\partial P}{\partial S}\right)_{0} \Delta V \Delta S-\left(\frac{\partial P}{\partial V}\right)_{0} \Delta V^{2}}{2 k T_{0}}=\frac{\Delta P \Delta V-\Delta T \Delta S}{2 k T_{0}} .
$$

Then, expression (2) is written in the form:

$$
w=A \exp \left[\frac{\Delta P \Delta V-\Delta T \Delta S}{2 k T}\right]
$$

(we do not write an index 0 in temperature, by $T$ is meant its equilibrium value).

In other case (constant volume, fluctuating number of particles) the change in entropy of environment during fluctuations in the subsystem should be written as

$$
\Delta S_{0}=\frac{\Delta U_{0}-\mu_{0} \Delta N_{0}}{T_{0}}=-\frac{\Delta U-\mu_{0} \Delta N}{T_{0}}
$$

and completely analogously to the first case, we obtain the expression

$$
w=A \exp \left[-\frac{\Delta T \Delta S+\Delta \mu \Delta N}{2 k T}\right] .
$$

There is another possibility of the separation of a subsystem from its environment when both volume and number of subsystem particles can fluctuate - separation by a physical boundary layer. The simplest examples are: drop of liquid in a vapor, gas bubble in a liquid, crystal in a melt, etc. In such cases one could obtain an expression, in which, contrary to (3) and (4), the exponent would be three-termed: $(\Delta P \Delta V-\Delta T \Delta S-\Delta \mu \Delta N) / 2 k T$. However, in the presence of the physical boundary between the environment and the subsystem, along with the volume fluctuations, the boundary surface fluctuations are essential. As this take place, new thermodynamic degrees of freedom (for example, capillary waves on the boundary surface, changing the facet of the crystal, etc.) appear, and the problem is significantly more complicated.

Let us find fluctuations of various thermodynamic quantities. First we choose $V, T$ as independent variables. Then,

$$
\Delta S=\left(\frac{\partial S}{\partial T}\right)_{V} \Delta T+\left(\frac{\partial S}{\partial V}\right)_{T} \Delta V=\frac{C_{V}}{T} \Delta T+\left(\frac{\partial P}{\partial T}\right)_{V} \Delta V,
$$




$$
\Delta P=\left(\frac{\partial P}{\partial T}\right)_{V} \Delta T+\left(\frac{\partial P}{\partial V}\right)_{T} \Delta V
$$

Substituting these expressions into the exponent of Eq. (3), we find that the terms of $\Delta V \Delta T$ are cancelling out and we obtain

$$
w=A \exp \left[-\frac{C_{V}}{2 k T^{2}}(\Delta T)^{2}+\frac{1}{k T}\left(\frac{\partial P}{\partial V}\right)_{T}(\Delta V)^{2}\right] .
$$

This expression splits into two factors that depend only on $\Delta T$ or $\Delta V$. In other words, volume and temperature fluctuations are statistically independent, and therefore

$$
\overline{\Delta T \Delta V}=0 .
$$

Comparing in succession either of the two decomposing factors (5) with the general formula for Gaussian distribution

$$
w(x) d x=\frac{1}{\sqrt{2 \pi \overline{x^{2}}}} \exp \left(-\frac{x^{2}}{2 \overline{x^{2}}}\right) d x
$$

we find such expressions for the mean squares of fluctuations in temperature and volume

$$
\overline{(\Delta T)^{2}}=\frac{k T^{2}}{C_{V}}, \quad \overline{(\Delta V)^{2}}=k T\left(-\frac{\partial V}{\partial P}\right)_{T} .
$$

The positivity of these quantities is provided by thermodynamic inequalities $C_{V}>0$ and $(-\partial P / \partial V)_{T}>0$.

Let us find the mean square of the energy fluctuations. We have

$$
\Delta H=\left(\frac{\partial H}{\partial V}\right)_{T} \Delta V+\left(\frac{\partial H}{\partial T}\right)_{V} \Delta T=\left[T\left(\frac{\partial P}{\partial T}\right)_{V}-P\right] \Delta V+C_{V} \Delta T .
$$

By squaring this expression and averaging, we get

$$
\overline{(\Delta H)^{2}}=k T\left(-\frac{\partial V}{\partial P}\right)_{T}\left[T\left(\frac{\partial P}{\partial T}\right)_{V}-P\right]^{2}+k T^{2} C_{V} .
$$

Considering (6), we can rewrite the condition (1) in the form

$$
\begin{gathered}
d \overline{(\Delta H)^{2}}=\left(d T \frac{\partial}{\partial T}+d V \frac{\partial}{\partial V}\right)\left[k T\left(-\frac{\partial V}{\partial P}\right)_{T}\left[T\left(\frac{\partial P}{\partial T}\right)_{V}-P\right]^{2}+k T^{2} C_{V}\right]=0, \text { or } \\
k T\left(-\frac{\partial V}{\partial P}\right)_{T}\left[T\left(\frac{\partial P}{\partial T}\right)_{V}-P\right]^{2}+k T^{2} C_{V}=\text { const } .
\end{gathered}
$$

The condition (7) describes a family of isolines of a constant level of energy fluctuations on $V T$ plane for this system. Alongside with these lines, there are isolines of constant level of internal energy 


$$
U=\bar{H}=\text { const }, d U=C_{V} d T+\left[T\left(\frac{\partial P}{\partial T}\right)_{V}-P\right] d V=0 .
$$

In general, the isolines being in different families intersect. The exceptions are the tangency points where the combining of the extreme properties of internal energy and its dispersion is observed. In the line of equal energies $d U=0$, extreme energy fluctuations are determined by the condition $d \overline{(\Delta H)^{2}}=0$ and vice versa. The combination of these two differential equations enables us to determine the curve with extreme fluctuation properties on $V T$ plane:

$$
\left\{\begin{array}{l}
C_{V} d T+\left[T\left(\frac{\partial P}{\partial T}\right)_{V}-P\right] d V=0 \\
\left(d T \frac{\partial}{\partial T}+d V \frac{\partial}{\partial V}\right)\left[k T\left(-\frac{\partial V}{\partial P}\right)_{T}\left[T\left(\frac{\partial P}{\partial T}\right)_{V}-P\right]^{2}+k T^{2} C_{V}\right]=0 .
\end{array}\right.
$$

Equation (8) can be considered as a system of homogeneous equations with unknown variables $d V$ and $d T$ with corresponding coefficients. A necessary criterion for the existence of a nontrivial solution of this system is equal-zero determinant composed of the coefficients of this system. This condition determines the equation of the line of extreme relative energy fluctuations, that is the equation of the line of supercritical transitions in the terms of variables $T$ and $V$ for the system under consideration:

$$
\left[C_{V} \frac{\partial}{\partial V}-\left[T\left(\frac{\partial P}{\partial T}\right)_{V}-P\right] \frac{\partial}{\partial T}\right]\left[k T\left(-\frac{\partial V}{\partial P}\right)_{T}\left[T\left(\frac{\partial P}{\partial T}\right)_{V}-P\right]^{2}+k T^{2} C_{V}\right]=0 .
$$

To compose equation (9), in each case it is necessary to know $(\partial S / \partial T)_{V}$ and $P=P(V, T)$. The latter value is nothing but thermal equation of state. To determine $(\partial S / \partial T)_{V}$ one can use the thermodynamic identity

$$
\frac{\partial}{\partial V}\left(\frac{\partial S}{\partial T}\right)_{V}=\left(\frac{\partial^{2} P}{\partial T^{2}}\right)_{V}
$$

from which it follows that

$$
C_{V}=T\left(\frac{\partial S}{\partial T}\right)_{V}=T \int\left(\frac{\partial^{2} P}{\partial T^{2}}\right)_{V} d V+C_{V}^{\mathrm{id}} .
$$

Equation (10) is quite accurate at some distance from the critical point, where the energy fluctuations are not very large, and can be used by the appropriate approximation. In the vicinity of the critical point where fluctuations are significant, the use of equation (10) needs to be clarified. The question arises about the validity of this equation at the critical point proper, which in its physical sense is the beginning of a supercritical line, where the liquid and gas states are separated.

Let us expand the condition (9): 


$$
\begin{gathered}
C_{V}\left[k T \frac{\partial}{\partial V}\left(-\frac{\partial V}{\partial P}\right)_{T}\left(T\left(\frac{\partial P}{\partial T}\right)_{V}-P\right)^{2}+2 k T\left(-\frac{\partial V}{\partial P}\right)_{T}\left[T\left(\frac{\partial P}{\partial T}\right)_{V}-P\right] \times\right. \\
\left.\times\left(T \frac{\partial^{2} P}{\partial T \partial V}+\left(-\frac{\partial P}{\partial V}\right)_{T}\right)\right]-\left[T\left(\frac{\partial P}{\partial T}\right)_{V}-P\right]\left(k\left(-\frac{\partial V}{\partial P}\right)_{T}\left[T\left(\frac{\partial P}{\partial T}\right)_{V}-P\right]^{2}+\right. \\
+2 k T C_{V}+2 k T\left(-\frac{\partial V}{\partial P}\right)_{T}\left[T\left(\frac{\partial P}{\partial T}\right)_{V}-P\right] T\left(\frac{\partial^{2} P}{\partial T^{2}}\right)_{V}+ \\
\left.+k T \frac{\partial}{\partial T}\left(-\frac{\partial V}{\partial P}\right)_{T}\left[T\left(\frac{\partial P}{\partial T}\right)_{V}-P\right]^{2}\right)=0 .
\end{gathered}
$$

With accounting the fact that

$$
\frac{\partial}{\partial V}\left(-\frac{\partial V}{\partial P}\right)_{T}=-\left(-\frac{\partial V}{\partial P}\right)_{T}^{2} \frac{\partial^{2} P}{\partial V \partial T} \text { and } \frac{\partial}{\partial T}\left(-\frac{\partial V}{\partial P}\right)_{T}=-\left(-\frac{\partial V}{\partial P}\right)_{T}^{2} \frac{\partial^{2} P}{\partial T \partial V},
$$

and rearranging the terms, we obtain

$$
\begin{array}{r}
{\left[T\left(\frac{\partial P}{\partial T}\right)_{V}-P\right]\left\{\left(-\frac{\partial V}{\partial P}\right)_{T} \frac{\partial^{2} P}{\partial T \partial V}\left[2 k T^{2} C_{V}+\left[T\left(\frac{\partial P}{\partial T}\right)_{V}-P\right]^{2}\left(-\frac{\partial V}{\partial P}\right)_{T}\right]+\right.} \\
+\left[T\left(\frac{\partial P}{\partial T}\right)_{V}-P\right]\left(-P\left(-\frac{\partial V}{\partial P}\right)_{T}^{2} \frac{\partial^{2} P}{\partial T \partial V}-k T C_{V}\left(-\frac{\partial V}{\partial P}\right)_{T}^{2}\left(-\frac{\partial^{2} P}{\partial V^{2}}\right)_{T}-\right. \\
\left.\left.-2 k T^{2}\left(-\frac{\partial V}{\partial P}\right)_{T}^{2}\left(\frac{\partial^{2} P}{\partial T^{2}}\right)_{V}\right)\right\}=0 .
\end{array}
$$

For systems, which thermal equation is linear in temperature, $\left(\partial^{2} P / \partial T^{2}\right)_{V}=0$ and heat capacity is $C_{V}=C_{V}^{\text {id }}$ because of (10). According to the Gibbs rule, $\left(\partial^{2} P / \partial T \partial V\right)=0$ at the critical point. This relation should also be fulfilled along the entire low-stability line where $(\partial P / \partial V)_{T}$ passes through a minimum as a function of $V$ and $T$. Considering this, we have

$$
\left[T\left(\frac{\partial P}{\partial T}\right)_{V}-P\right]^{2}\left[-k T C_{V}\left(-\frac{\partial V}{\partial P}\right)_{T}^{2}\left(-\frac{\partial^{2} P}{\partial V^{2}}\right)_{T}\right]=0 \quad \text { or } \quad\left(-\frac{\partial^{2} P}{\partial V^{2}}\right)_{T}=0 .
$$

And this is the equation of the line of supercritical transitions according to V.K. Semenchenko [12].

A similar result can be obtained for magnets if using the analogy of liquid and magnetic systems.

Let us now consider this situation in the general case of a simple one-component system, which is under thermodynamic forces $T$ and $X$ [18]. Determinant of stability is 


$$
D=\frac{\partial(T, X)}{\partial(S, x)}=\left|\begin{array}{ll}
\left(\frac{\partial T}{\partial S}\right)_{x} & \left(\frac{\partial T}{\partial x}\right)_{S} \\
\left(\frac{\partial X}{\partial S}\right)_{x} & \left(\frac{\partial X}{\partial x}\right)_{S}
\end{array}\right|=\left(\frac{\partial T}{\partial S}\right)_{x}\left(\frac{\partial X}{\partial x}\right)_{S}-\left(\frac{\partial T}{\partial x}\right)_{S}^{2} .
$$

Then, as mentioned previously, the following condition must be fulfilled on the supercritical transition line:

$$
d D=\left(\frac{\partial D}{\partial S}\right)_{x} d S+\left(\frac{\partial D}{\partial x}\right)_{S} d x=0 \quad \text { or } \quad\left(\frac{\partial D}{\partial S}\right)_{x}=0, \quad\left(\frac{\partial D}{\partial x}\right)_{S}=0 .
$$

With accounting Eq. (12), the last two equations give:

$$
\begin{aligned}
& \left(\frac{\partial^{2} T}{\partial S^{2}}\right)_{x}\left(\frac{\partial X}{\partial x}\right)_{S}+\left(\frac{\partial T}{\partial S}\right)_{x} \frac{\partial^{2} X}{\partial x \partial S}-2\left(\frac{\partial T}{\partial x}\right)_{S} \frac{\partial^{2} T}{\partial x \partial S}=0 \\
& \frac{\partial^{2} T}{\partial S \partial x}\left(\frac{\partial X}{\partial x}\right)_{S}+\left(\frac{\partial T}{\partial S}\right)_{x}\left(\frac{\partial^{2} X}{\partial x^{2}}\right)_{S}-2\left(\frac{\partial T}{\partial x}\right)_{S}\left(\frac{\partial^{2} T}{\partial x^{2}}\right)_{S}=0 .
\end{aligned}
$$

This system of equations holds if

$$
\left(\frac{\partial^{2} T}{\partial S^{2}}\right)_{x}=\frac{\partial^{2} T}{\partial S \partial x}=\frac{\partial^{2} X}{\partial x \partial S}=\left(\frac{\partial^{2} X}{\partial x^{2}}\right)_{S}=\left(\frac{\partial^{2} T}{\partial x^{2}}\right)_{S}=0
$$

Putting $X=-P, H, E$ and $x=V, M, \mathscr{P}$ (where $P$ is pressure, $H$ and $E$ are intensities of, respectively, magnetic and electric fields, $V$ is volume, $M$ is magnetization and $\mathscr{P}$ is electric polarization), we obtain the quasi-spinodal equation for liquid, magnetic, and dielectric systems.

\section{Conclusions}

In this paper we considered thermodynamic stability of a simple one-component system (liquid and generalized one, which is under thermodynamic forces $T$ and $X$ ) above the critical point. When the temperature exceeds the critical temperature, the system passes through a region of lowered stability. This leads to the increase of fluctuations of energy and external parameters of the system. From the point of view of thermodynamic stability, this indicates the existence of a continuous supercritical transition between supercritical mesophases. We studied the behavior of thermodynamic parameters of the system in the supercritical area of lowered stability both from viewpoints of thermodynamic stability theory and quasi-thermodynamic theory of fluctuations and obtained the equation of the line of supercritical transition (quasi-spinodal).

\section{References}

1. Kagoshima S. Critical light scattering in ${ }^{4} \mathrm{He}$ near the gas-liquid critical point / S. Kagoshima, K. Ohbayashi, A. Ikushima // Journal of Low Temperature Physics. 1973. - Vol. 11, Issue 5-6. - P. $765-774$.

2. Bondarenko G.V. Vliyanie temperatury i davleniya na kolebatelno-vraschatelnye spektry nadkriticheskoi vody / G.V. Bondarenko, Yu.Ye. Gorbatyi, V.M. Edelshtein // Doklady AN SSSR. - 1974. - Vol. 214, No. 2. - P. 365 - 368. 
3. Yerokhin N.F. Eksperimentalnoie issledovaniie skorosti ultrazvuka v kriticheskoi oblasti vody / N.F. Yerokhin, B.I. Kalianov // Teplofizika vysokikh temperatur. - 1979. Vol. 17, Issue 2. - P. 290 - 298.

4. Anisimov M.A. Kriticheskiie yavleniya v zhidkostiakh i zhidkikh kristallakh / M.A. Anisimov. - Moscow: Nauka, 1987. - 272 p.

5. Anisimov M.A. Termodinamika kriticheskogo sostoyaniya individualnykh veshchestv / M.A. Anisimov, V.A. Rabinovich, V.V. Sychev. - Moscow: Energoizdat, 1990. - $190 \mathrm{p}$.

6. Kostko A.F. Criticality in aqueous solutions of 3-methylpyridine and sodium bromide / A.F. Kostko, M.A. Anisimov, J.V. Sengers // Physical Review E. - 2004. Vol. 70, Issue 2. - P. 026118-1 - 026118-11.

7. Yerokhin N.F. Osobennosti ultraakusticheskikh izmerenij v liogkoj i tyazhioloj vodie v kriticheskoj oblasti / N.F. Yerokhin, V.I. Kompaniets, Yu.V. Leonov // Vestnik Taganrogskogo instituta im. A.P. Chekhova. Yestestvennye nauki. Fizika. -2006. - No. 1. - P. $134-138$.

8. Bulavin L.A. Krytychni yavyshcha rozsharuvannya $\mathrm{v}$ ridynakh na Zemli ta $\mathrm{v}$ kosmosi / L.A. Bulavin, Yu.O. Plevachuk, V.M. Sklyarchuk. - Kyiv: Naukova dumka, 2011. $-280 \mathrm{p}$.

9. Bulavin L.A. Neitronna diagnostyka ridkoho stanu rechovyny / L.A. Bulavin. Chornobyl: Instytut problem bezpeky AES, 2012. - 532 p.

10. Soldatova E.D. Variety of critical state nature manifestations / E.D. Soldatova // Condensed Matter Physics. - 1999. - Vol. 2, No. 4 (20). - P. 603 - 616.

11. Soldatova E.D. Thermodynamic stability of equilibrium system and critical state / E.D. Soldatova // Journal of Molecular Liquids. - June 2005. - Vol. 120, Issue 1-3. - P. $47-50$.

12. Semenchenko V.K. Termodinamicheskaya ustojchivost i fazovye perehody v kristallakh / V.K. Semenchenko // Kristallografiya. - 1964. - Vol. 9, No. 5. - P. 611 621.

13. Vonsovskii S.V. Magnetizm. Magnitnye svoistva dia-, para-, ferro-, antiferro-, I ferrimagnetikov / S.V. Vonsovskii. - Moscow: Nauka, 1971. - 1032 p.

14. Ohta H. Magnetic study of $\mathrm{SmCoAsO}$ showing a ferromagneticantiferromagnetic transition / H. Ohta, C. Michioka, A. Matsuo, K. Kindo, K. Yoshimura // Physical Review B. - 2100. - Vol. 82, Issue 5. - P. 054421-1 - 054421-8.

15. Landau L.D. Statisticheskaya fizika. Part 1 / L.D. Landau, Ye.M. Lifshits. Moscow: Nauka, 1976. - 584 p. - (Seria "Teoreticheskaya fizika"; Vol. 5).

16. Rudoi Yu.G. Termodynamicheskie fluctuatsii $\mathrm{v}$ podkhodakh Gibbsa i Einshteina / Yu.G. Rudoi, A.D. Sukhanov // Uspekhi fizicheskikh nauk. - 2000. - Vol. 170, No. 12. - P. 1265 - 1296.

17. Soldatova E.D. Stability conditions for the basic thermodynamic potentials and the substantiation of the phase diagrams / E.D. Soldatova // Journal of Molecular Liquids. - 2006. - Vol. 127, Issue 1-3. - P. 99 - 101.

18. Galdina A.N. Rivnyannya linii znyzhenoji stijkosti dlya modeli Liba / A.N. Galdina // Visnyk Dnipropetrovskogo universitetu. Serija Fizika. Radioelektronika. - 2007. - Vol. 14, Issue 12/1. - P. 68 - 70. 